\title{
ESCRT machinery plays a role in microautophagy in yeast
}

\author{
Shamsul Morshed ${ }^{1}$, Most Naoshia Tasnin ${ }^{1}$ and Takashi Ushimaru ${ }^{1,2^{*}}$ (i)
}

\begin{abstract}
Background: Microautophagy, which degrades cargos by direct lysosomal/vacuolar engulfment of cytoplasmic cargos, is promoted after nutrient starvation and the inactivation of target of rapamycin complex 1 (TORC1) protein kinase. In budding yeast, microautophagy has been commonly assessed using processing assays with green fluorescent protein (GFP)-tagged vacuolar membrane proteins, such as Vph1 and Pho8. The endosomal sorting complex required for transport (ESCRT) system is proposed to be required for microautophagy, because degradation of vacuolar membrane protein Vph1 was compromised in ESCRT-defective mutants. However, ESCRT is also critical for the vacuolar sorting of most vacuolar proteins, and hence reexamination of the involvement of ESCR $T$ in microautophagic processes is required.
\end{abstract}

Results: Here, we show that the Vph1-GFP processing assay is unsuitable for estimating the involvement of ESCRT in microautophagy, because Vph1-GFP accumulated highly in the prevacuolar class E compartment in ESCRT mutants. In contrast, GFP-Pho8 and Sna4-GFP destined for vacuolar membranes via an alternative adaptor protein-3 (AP-3) pathway, were properly localized on vacuolar membranes in ESCRT-deficient cells. Nevertheless, microautophagic degradation of GFP-Pho8 and Sna4-GFP after TORC1 inactivation was hindered in ESCRT mutants, indicating that ESCRT is indeed required for microautophagy after nutrient starvation and TORC1 inactivation.

Conclusions: These findings provide evidence for the direct role of ESCRT in microautophagy induction.

Keywords: AP-3 pathway, ESCRT, Microautophagy, Pho8, Vph1, VPS pathway

\section{Background}

In microautophagy, cytoplasmic cargos are directly engulfed by lysosomal/vacuolar membranes, sorted into the vacuolar lumen, and degraded [1-3]. Because vacuolar membrane proteins together with vacuolar membranes are degraded in the vacuole in the course of microautophagy, overall microautophagic flux is estimated using green fluorescent protein (GFP)-tagged vacuolar transmembrane proteins, Vph1 (a subunit of the vacuolar-ATPase V0 domain) and Pho8 (vacuolar alkaline phosphatase) in the budding yeast Saccharomyces

\footnotetext{
* Correspondence: ushimaru.takashi@shizuoka.ac.jp

${ }^{1}$ Graduate School of Science and Technology, Shizuoka University, Ohya 836, Suruga-ku, Shizuoka 422-8021, Japan

${ }^{2}$ Department of Science, Shizuoka University, Ohya 836, Suruga-ku, Shizuoka 422-8021, Japan
}

cerevisiae [4]. When Vph1-GFP and GFP-Pho8 are incorporated into the vacuolar lumen by microautophagy, Vph1 and Pho8, but not the stable GFP moiety, are degraded by vacuolar proteases, generating free GFP, which is detectable by immunoblotting. Nutrient starvation or inactivation of target of rapamycin complex 1 (TORC1) protein kinase evokes microautophagy [4-6].

The endosomal sorting complex required for transport (ESCRT) system was originally identified as being involved in the formation of intraluminal vesicles within multivesicular bodies [7-9]. The ESCRT-0 complex binds to ubiquitinated proteins on endosomal membranes and recruits the ESCRT-I and -II complexes, eliciting the assembly of ESCRT-III, which promotes the invagination, constriction, and abscission of endosomal membranes. In microautophagy, carbon starvation after 
diauxic shift or TORC1 inactivation promotes recruitment of ESCRT-0 onto vacuolar membranes $[4,10]$. Furthermore, free GFP production from Vph1-GFP after diauxic shift was significantly impeded in cells lacking ESCRT-0, -I, -II or -III, inferring a model where ESCR $\mathrm{T}$ is required for the deformation of the vacuolar membrane and, thus, also microautophagy [4]. However, most proteins destined for vacuoles pass through late endosomes, named the vacuolar protein sorting (VPS) pathway (or the carboxypeptidase Y pathway), and hence they are trapped in the class E compartment in ESCRT mutants $[11,12]$. This suggested a possibility that Vph1GFP is not located properly on vacuolar membranes during microautophagy induction, thereby causing a reduction in the autophagic degradation of Vph1-GFP. Thus, it remains unclear whether or not ESCRT is directly necessary for microautophagic processes on vacuolar membranes.

We found that Vph1 accumulated in the perivacuolar class E compartment in vps $27 \Delta$ cells defective in ESCRT0 , but we did not further investigate the involvement of ESCRT in sorting of Vph1-GFP and GFP-Pho8 [5]. In addition, we also found that loss of ESCRT compromised microautophagic degradation of GFP-Pho8 after rapamycin treatment, but we did not check whether Pho8 was properly localized on vacuolar membranes in ESCRT mutant cells [10]. Here, we show that Pho8 destined for vacuolar membranes via an alternative adaptor protein-3 (AP3) pathway, but not Vph1, is correctly targeted to vacuolar membranes even in ESCRT mutants and after TORC1 inactivation. Nevertheless, free GFP production from GFPPho8 after TORC1 inactivation was hindered in ESCRT mutants. These findings demonstrate that the ESCRT machinery is genuinely necessary for microautophagic processes. Another AP-3 pathway-dependent vacuolar membrane protein Sna4 confirmed this idea.

\section{Results}

Vph1-GFP is an unsuitable tool to assess the involvement of ESCRT in microautophagy

To assess microautophagic flux/activity using a processing assay with a GFP-tagged vacuolar membrane protein, its proper localization in vacuolar membranes is prerequisite. First, we assessed whether Vph1 is correctly localized in cells defective in each ESCRT complex, ESCRT-0 to -III, both in normal (nutrient-rich and TORC1 active) conditions and during microautophagy induction after TORC1 inactivation. We found that ESCRT-lacking mutant cells, with mutations in Vps27 (ESCRT-0), Vps28 (ESCRT-I), Vps36 (ESCRT-II) or Vps24 (ESCRT-III), showed massive accumulation of Vph1-GFP in the perivacuolar class E compartment in normal (nutrient-rich and TORC1 active) conditions (Fig. 1, control) as described previously [5, 13-16]. This clearly confirmed that $\mathrm{Vph} 1$ is delivered to the vacuolar membrane via the VPS pathway. The aberrant accumulation of Vph1-GFP still remained during microautophagy induction after rapamycin treatment (Fig. 1, +Rap). Namely, Vph1-GFP did not properly reach the vacuolar surface in ESCRT-deficient cells regardless of TORC1 activity. This demonstrated that the Vph1-GFP processing assay is not suitable to evaluate whether ESCRT is directly implicated in microautophagic processes, although Oku et al. proposed it based on results obtained using this assay [4].

\section{GFP-Pho8 is a suitable marker to evaluate the involvement of ESCRT in microautophagy}

Next, we similarly assessed GFP-Pho8. In contrast to most vacuolar proteins trafficked via the VPS pathway, a small number of proteins including the vacuolar membrane alkaline phosphatase (ALP) Pho8 are transported directly from the Golgi to the vacuolar surface, even in class E mutants, via the adaptor protein-3 (AP-3) pathway (or the ALP pathway) [14, 17-19]. We also observed that GFP-Pho8 was appropriately located on vacuolar membranes in ESCRT mutants in normal conditions (Fig. 2, control). Furthermore, its vacuolar localization was not lost during microautophagy induction after cells were treated with rapamycin (Fig. 2, +Rap). Thus, GFPPho8 is properly localized on the vacuolar membrane in ESCRT mutants regardless of TORC1 activity. We concluded that GFP-Pho8 is a suitable marker to address the involvement of ESCRT in microautophagy.

\section{ESCRT is required for microautophagy after TORC1 inactivation}

In a parallel study, we recently reported that free GFP generation from GFP-Pho8 after treatment with rapamycin was massively compromised in cells defective in

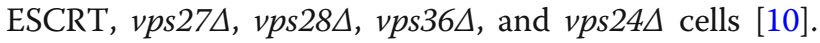
Given the proper localization of GFP-Pho8 on vacuolar membranes in these ESCRT mutants, these observations indicated that ESCRT is required for microautophagy itself. To further confirm this we subjected cells to nitrogen starvation, which is a natural condition where TORC1 is inactivated. GFP-Pho8 still showed proper distribution on the vacuolar surface after nitrogen starvation even in ESCRT mutants (Fig. 3a). Autophagic degradation of GFP-Pho8 after nitrogen starvation was mildly reduced in these ESCRT mutants (Fig. 3b) (see "Discussion"). These findings confirmed the idea that ESCRT is indeed required for proper microautophagic induction after TORC1 inactivation.

Search for other appropriate markers of microautophagy Thus, proteins destined for vacuolar membranes via the AP-3 pathway (the ALP pathway) have potential as 


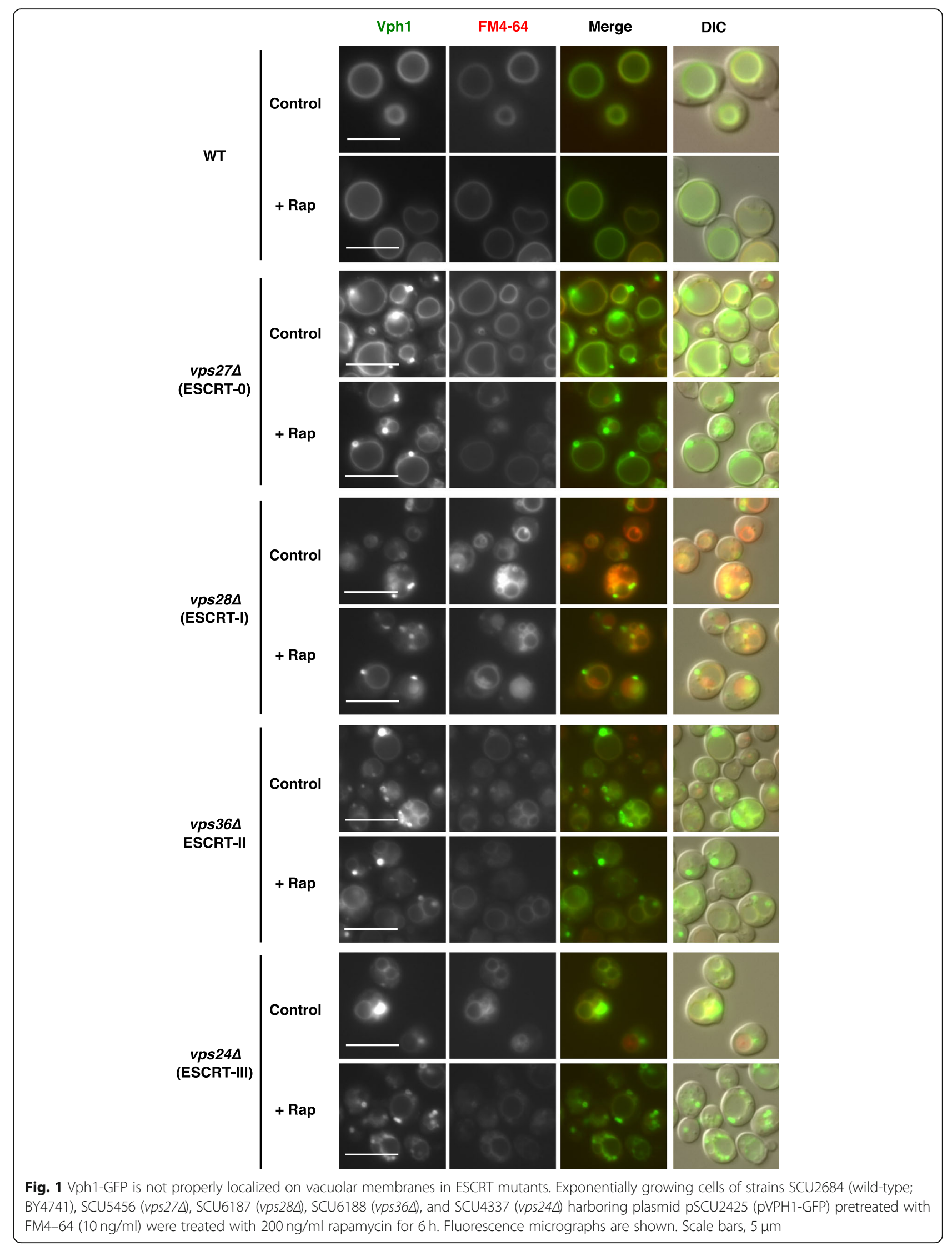




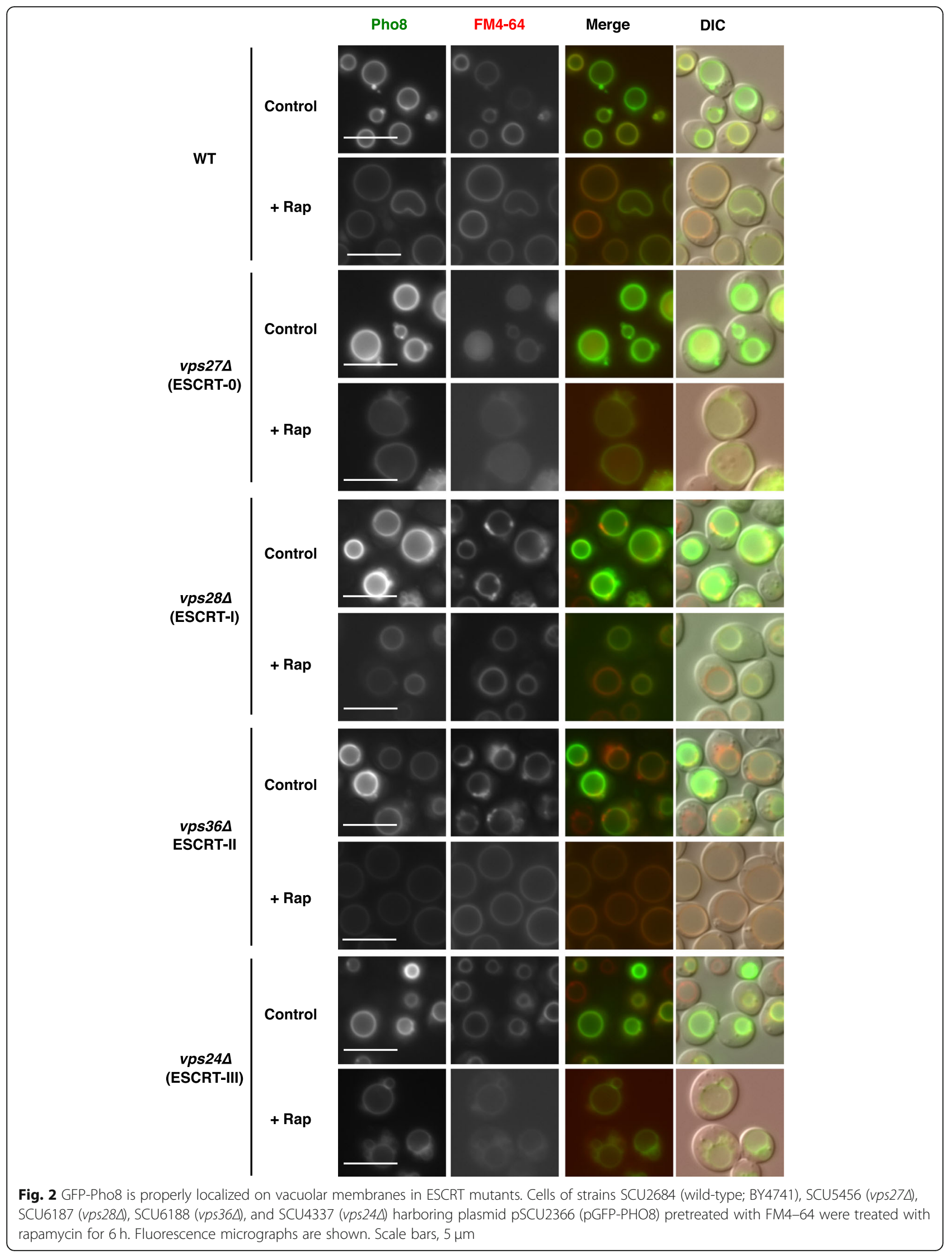




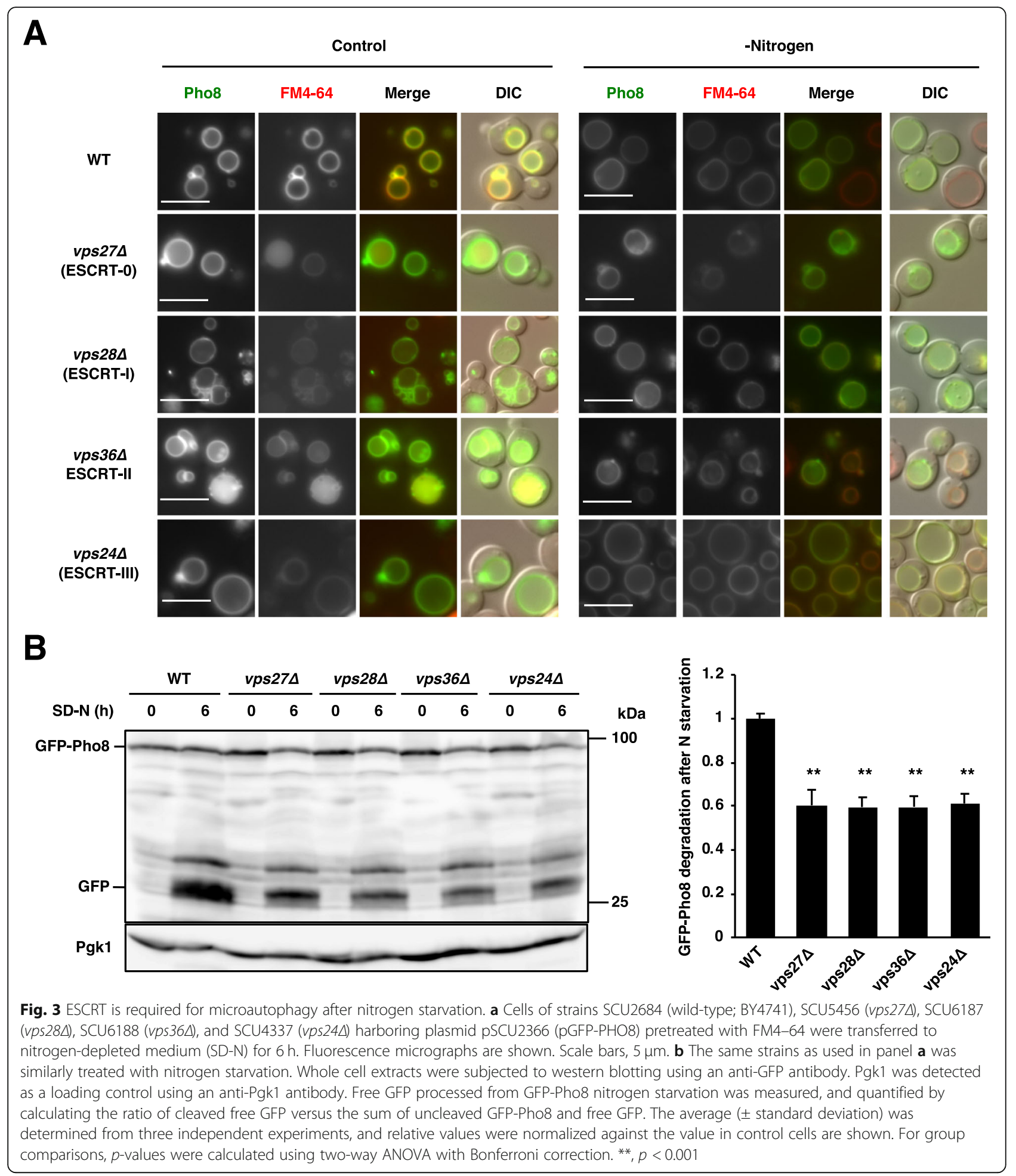

appropriate markers to investigate ESCRT-mediated microautophagy. We assessed this using the following vacuolar membrane proteins targeted via the AP-3 pathway: Nyv1 (vacuolar v-SNARE), Yck3 (casein kinase) and Sna4 (protein of unknown function) [20-22]. We found that Nyv1-GFP was not clearly distributed on vacuolar membranes even in wild-type cells (Additional file 1: Fig. S1a). By contrast, clear localization of Yck3-GFP on vacuolar membranes was found in wild-type cells in the absence or presence of rapamycin (Additional file 1: Figs. S1a, b). However, unlike Vph1-GFP and GFP-Pho8, Yck3-GFP did not generate free GFP after rapamycin 
treatment, although Yck3-GFP was almost lost (Additional file 1: Fig. S1c). This suggested that Yck3 is degraded outside vacuoles (see "Discussion"). Thus, Nyv1GFP and Yck3-GFP were not suitable for the assessment microautophagic flux. Finally, we found that Sna4-GFP was located on vacuolar membranes in wild-type cells in the absence and presence of rapamycin and that similar vacuolar localization was maintained in ESCRT mutants, although Sna4-GFP signals on the vacuolar membranes were largely lost after rapamycin treatment (Fig. 4, Additional file 1: Fig. S2). Sna4-GFP produced free GFP in wild-type cells after rapamycin treatment (Fig. 5, see also Additional file 1: Fig. S3), which was repressed in vps274 cells, similar to GFP-Pho8 [10]. In addition, free GFP generation from Sna4-GFP after rapamycin treatment was also reduced in vps $28 \Delta$, vps $36 \Delta$, and vps $24 \Delta$ cells, although uncharacterized protein bands accumulated additionally in wild-type and the mutant cells. These findings confirmed the hypothesis that ESCRT is required for proper microautophagy induction.

\section{Discussion}

In this study, we showed that GFP-Pho8 and Sna4-GFP are suitable tools to evaluate the involvement of ESCRT in microautophagic processes on vacuolar membranes and that ESCRT is genuinely required for this process after TORC1 inactivation. In contrast, Vph1-GFP is not suitable for use in this assay, because Vph1-GFP was not correctly localized on the vacuolar membranes in ESCR T mutants.

We tested whether other vacuolar membrane proteins sorted via the AP-3 pathway (Nyv1 and Yck3) are suitable as markers of microautophagic flux. However, they were found not to be useable markers for microautophagy assays. In the course of this study, we noticed that the vacuolar membrane protein Yck3 was not degraded in the vacuole after TORC1 inactivation, although microautophagy is induced: there was no accumulation of free GFP (a marker of vacuolar degradation) from Yck3-GFP after rapamycin treatment, although Yck3GFP was lost. It is plausible that Yck3 is degraded by proteasomes in the cytoplasm after TORC1 inactivation. The armadillo repeat protein Vac8 is localized on the vacuolar membrane, and has multiple functions including vacuole inheritance [23, 24]. No free GFP production from Vac8-GFP appeared after rapamycin treatment (Morshed et al., unpublished data). These findings indicated that some, but not all, vacuolar membrane proteins are degraded by microautophagy after TORC1 inactivation. It is an interesting open question how some vacuolar membrane proteins escape from microautophagic degradation after TORC1 inactivation. Furthermore, Sna4-GFP was lost with no clear free GFP accumulation after rapamycin treatment in vps27A cells (Fig. 5), indicating that Sna4 was degraded outside vacuoles in specific situations. These findings imply that some vacuolar membrane proteins are degraded by multiple pathways after TORC1 inactivation.

In the case of nitrogen starvation, loss of ESCRT mildly reduced nitrogen starvation-induced microautophagy (Fig. 3b). This suggested a possibility that ESCRTindependent microautophagy is promoted after nitrogen starvation. Alternatively, a microautophagy-independent pathway might mediate degradation of vacuolar membrane proteins under these conditions. It has been reported that ESCRT-independent homotypic vacuolevacuole fusion mediates degradation of vacuolar membrane proteins (the intraluminal fragment pathway) [25].

This study concluded that ESCRT is indeed involved in microautophagic processes in budding yeast. However, the molecular mechanism of ESCRT-mediated microautophagy is still largely unknown. This study provides a caution for the selection of markers to assess microautophagic flux. To evaluate microautophagic activity in mutants using GFP-tagged vacuolar membrane proteins, confirmation of their proper localization on vacuolar membranes is prerequisite. It is suggested that microautophagy is involved in protein homeostasis in mammalian cells [26]. However, the molecular mode and physiological roles of mammalian microautophagy are still largely unknown $[27,28]$. This study is helpful for dissection of the mechanism and physiological meaning of microautophagy not only in budding yeast but also other organisms including humans.

\section{Conclusions}

Microautophagic degradation of GFP-Pho8 and Sna4GFP after TORC1 inactivation was hindered in ESCRTdeficient cells, although they were properly sorted onto vacuolar membranes. This fact indicates that ESCRT is indeed required for microautophagy after nutrient starvation and TORC1 inactivation.

\section{Methods}

\section{Strains and media}

The S. cerevisiae strains and plasmids are listed in Additional files: Tables S1 and S2, respectively. Glucosecontaining YPAD (YPD containing 0.01\% adenine) and synthetic minimal medium (SD) complemented with the appropriate nutrients for plasmid maintenance were prepared using standard methods. For assessment of microautophagy after rapamycin treatment, when cells harbored plasmids, cells were precultured in SD medium with the appropriate nutrients, and then cultured in YPAD, followed by rapamycin treatment. For nitrogenstarvation experiments, cells were transferred into SD-N medium without ammonium sulfate. 


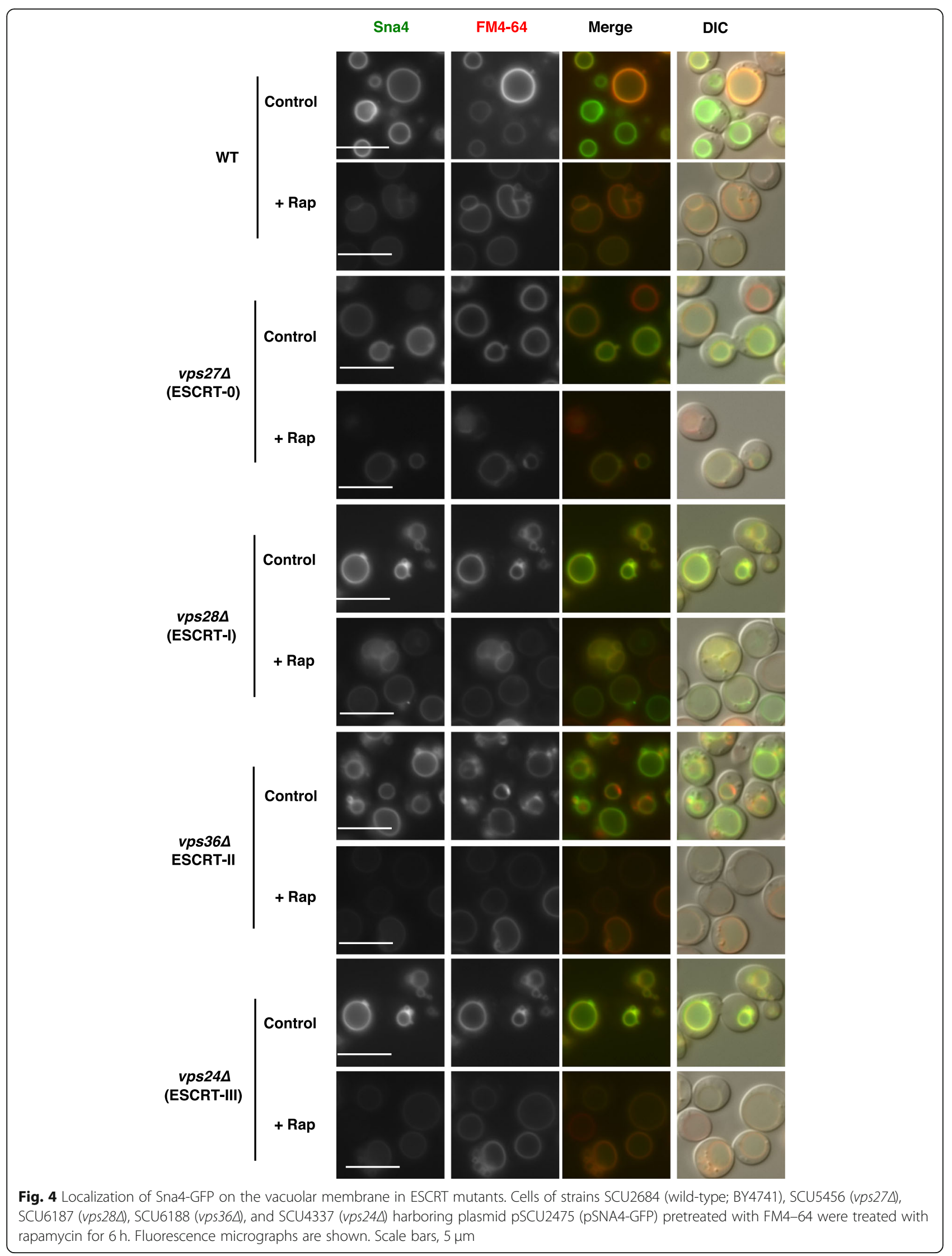



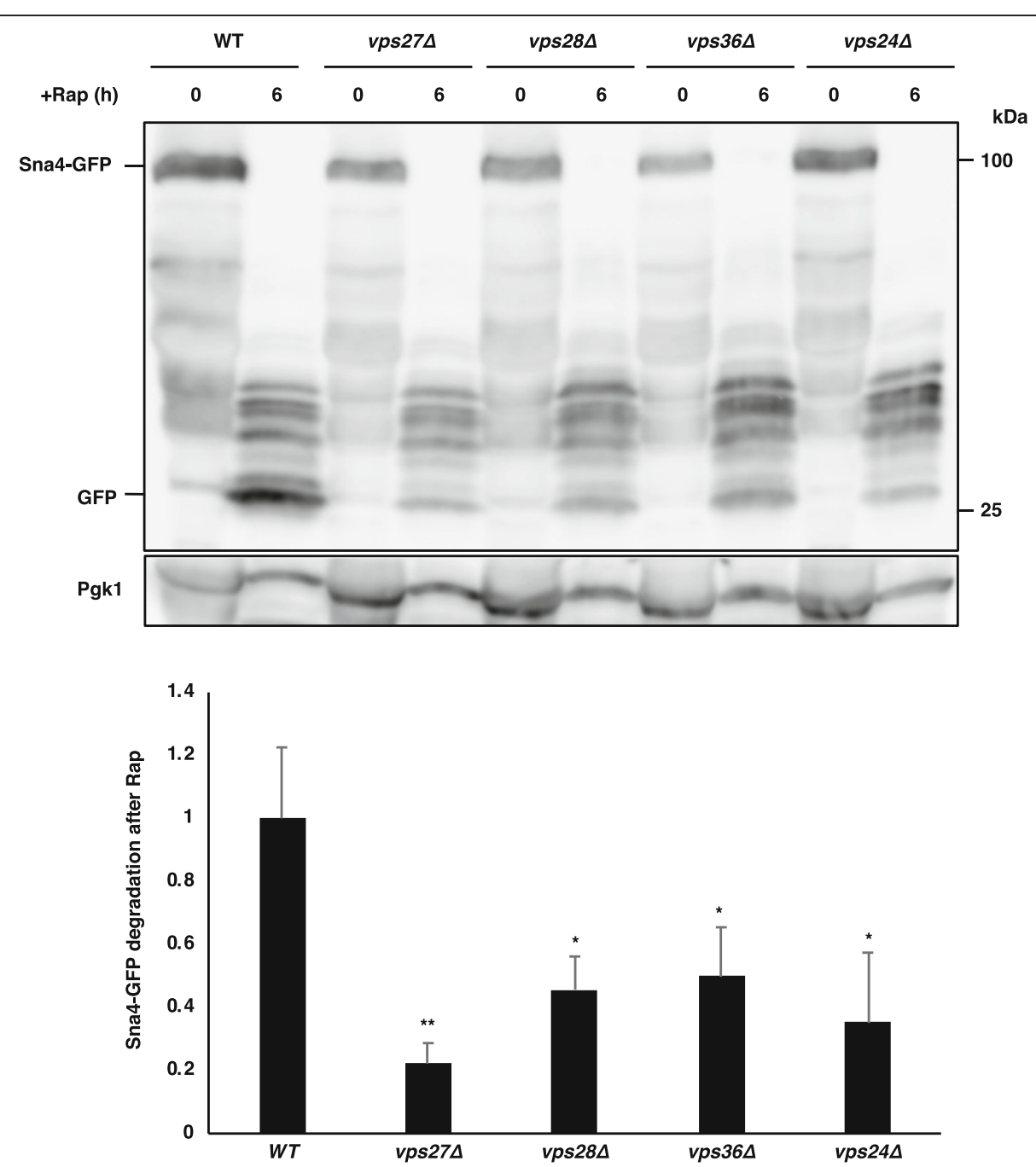

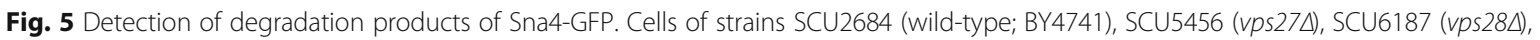
SCU6188 (vps364), and SCU4337 (vps244) harboring plasmid pSCU2475 (pSNA4-GFP) were treated with rapamycin for 6 h. Whole cell extracts were subjected to western blotting using the anti-GFP antibody. To assess microautophagic flux, the ratios of cleaved free GFP versus Pgk1 were calculated and the values relative to that in wild-type cells are shown. The average and standard deviation were determined from three independent experiments, and relative values were normalized against the value in control cells are shown. $p$-values were calculated using twoway ANOVA with Bonferroni correction. ${ }^{*}, p<0.05 ;{ }^{*}, p<0.005$

\section{Western blotting analysis}

Exponentially growing cells were used and proteins were extracted using a post-alkaline extraction method in accordance with a previous report [29]. We used the following antibodies: anti-GFP mouse monoclonal antibody (Santa Cruz, Cat\#sc-9996; RRID: AB_627695) and antiPgk1 mouse monoclonal antibody (Abcam, Cat\# ab113687; RRID: AB_10861977). All western blotting experiments were performed independently at least three times to confirm the reproducibility of the results. Relative protein amounts were measured using ImageJ software. To assess microautophagic flux, free GFP processed from of GFP-Pho8 after rapamycin treatment or nitrogen starvation was measured and quantified by calculating the ratio of cleaved free GFP versus the sum of uncleaved GFP-fused proteins and free GFP, and the values relative to that in wild-type cells are shown, as described previously [30]. In the case of Sna4-GFP, uncleaved Sna4-GFP was almost lost after rapamycin treatment. Therefore, to assess microautophagic flux using Sna4-GFP, the ratios of cleaved free GFP versus Pgk1 were calculated, and the values relative to that in wild-type cells are shown. The average and standard deviation were determined for each sample from three independent experiments, and relative values normalized against the value in control cells are shown. For group comparison, data analysis was performed with the software GraphPad Prism (v. 8.1.2) followed by two-way ANOVA (or mixed model) with Bonferroni correction for multiple comparisons in statistical hypothesis testing. 


\section{Microscope observations}

Exponentially growing cells were used for experiments. Cells were stained with the vacuolar membrane dye FM4-64 $(10 \mathrm{ng} / \mathrm{ml})$ for $1 \mathrm{~h}$, washed to remove free dye and further incubated at least for $1.5 \mathrm{~h}$ prior to treatment with rapamycin or nitrogen starvation. Cell, GFP, and FM4-64 images were captured using a Carl Zeiss Axio Imager M1 microscope with a cooled CCD camera (Carl Zeiss AxioCam MRm) [10]. All microscope observations were performed independently at least three times to confirm the reproducibility of the results. For quantification of intensities of Sna4-GFP signals on the vacuolar membrane, we used a microscopy-equipped software (Carl Zeiss AxioVision) according to a previous study [31].

\section{Statistical analysis}

Statistical analysis was performed using Microsoft Excel and GraphPad Prism 8 software.

\section{Supplementary information}

Supplementary information accompanies this paper at https://doi.org/10. 1186/s12860-020-00314-W.

Additional file 1 Fig. S1. Characterizations of $Y_{c k 3}$ and Nyv1, vacuolar membrane proteins sorted via the AP-3 pathway. a Fluorescence micrographs of cells of strains SCU6206 (YCK3-GFP) and SCU6207 (NYV1-GFP). Fluorescence micrographs of cells are shown. Scale bars, $5 \mu \mathrm{m}$. b Cells of strains SCU6206 (YCK3-GFP) were treated with rapamycin for $6 \mathrm{~h}$. Scale bars, $5 \mu \mathrm{m}$. c Cells of strain SCU6206 (YCK3-GFP) were treated with rapamycin for $6 \mathrm{~h}$. Whole cell extracts were subjected to western blotting using the anti-GFP antibody. Fig. S2. Intensity of Sna4-GFP signals on the vacuolar membranes (Related to Fig. 4). a Illustration for measurement of intensities of fluorescent signals of Sna4-GFP on vacuolar membranes. $\mathbf{b}$ Examples for measurement of the intensities of GFP on the vacuolar membrane using a captured cell images. Cells expressing a GFPtagged vacuolar membrane protein were treated with rapamycin for $6 \mathrm{~h}$. c The intensities of Cells of strains SCU2684 (wild-type; BY4741), SCU5456

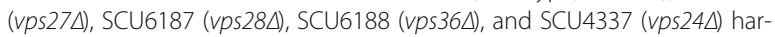
boring plasmid pSCU2475 (pSNA4-GFP) signals on vacuolar membranes in non-treated control and rapamycin-treated cells are shown in the box plot with dots. Sample sizes are 15. p-values were calculated using twoway ANOVA test (or mixed model) with Bonferroni correction. ${ }^{* *}, p<$ 0.0001. $p$ values between intensities in wild-type and each ESCRT mutant were higher than 0.05 before and after rapamycin treatment. Fig. S3. Sna4-GFP signals before and after rapamycin treatment (Related to Fig. 5). Cells of strain SCU2684 (wild-type) harboring plasmid pSCU2475 (pSNA4-GFP) were treated with rapamycin for $6 \mathrm{~h}$. Whole cell extracts were subjected to western blotting using anti-GFP antibody. For the control, wild-type cells without pSNA4-GFP were used. Fig. S4. Independent western blot images with quantifications (Related to Fig. 3b). Fig. S5. Independent western blot images with quantifications (Related to Fig. 5).

Additional file $\mathbf{2}$ Table S1. Yeast strains used in this study.

Additional file $\mathbf{3}$ Table S2. Plasmids used in this study.

\section{Abbreviations}

ALP: Alkaline phosphatase; AP-3: Adaptor protein-3; ESCRT: Endosomal sorting complex required for transport; GFP: Green fluorescent protein; TORC1: Target of rapamycin complex 1; VPS: Vacuolar protein sorting

\section{Acknowledgments}

We thank Muhammad Arifur Rahman for providing preliminary findings and laboratory members of TU for helpful discussions.

\section{Authors' contributions}

TU designed the project and experiments. SM mainly conducted the experiments. MNT conducted some experiments. SM and MNT analyzed the data. TU mainly wrote the paper and SM helped to write the paper. All authors read and approved the final manuscript.

\section{Funding}

This work was supported in part by the Japan Society for the Promotion of Science (JSPS) (grant No. 18 K06212 and 20H05317) and Institute for Fermentation, Osaka (IFO) (grant No. 2020-2-055). The funding bodies played no role in the design of the study, collection, analysis, interpretation of data, or writing of the manuscript.

\section{Availability of data and materials}

Data and materials used in the current study are available from the corresponding authors on request.

\section{Ethics approval and consent to participate}

Not applicable.

\section{Consent for publication}

Not applicable.

\section{Competing interests}

The authors declare that they do not have any competing interests

Received: 26 March 2020 Accepted: 1 October 2020

Published online: 07 October 2020

\section{References}

1. Muller O, Sattler T, Flotenmeyer M, Schwarz H, Plattner H, Mayer A. Autophagic tubes: vacuolar invaginations involved in lateral membrane sorting and inverse vesicle budding. J Cell Biol. 2000;151:519-28.

2. Sattler T, Mayer A. Cell-free reconstitution of microautophagic vacuole invagination and vesicle formation. J Cell Biol. 2000;151:529-38.

3. Kunz JB, Schwarz H, Mayer A. Determination of four sequential stages during microautophagy in vitro. J Biol Chem. 2004;279:9987-96.

4. Oku M, Maeda Y, Kagohashi Y, Kondo T, Yamada M, Fujimoto T, Sakai Y. Evidence for ESCRT- and clathrin-dependent microautophagy. J Cell Biol. 2017:216:3263-74.

5. Rahman MA, Terasawa M, Mostofa MG, Ushimaru T. The TORC1-Nem1/ Spo7-Pah1/lipin axis regulates microautophagy induction in budding yeast. Biochem Biophys Res Commun. 2018:504:505-12.

6. Hatakeyama R, Peli-Gulli MP, Hu Z, Jaquenoud M, Garcia Osuna GM, Sardu A, Dengjel J, De Virgilio C. Spatially distinct pools of TORC1 balance protein homeostasis. Mol Cell. 2019;73:325-38 e8.

7. Williams RL, Urbe S. The emerging shape of the ESCRT machinery. Nat Rev Mol Cell Biol. 2007:8:355-68.

8. Saksena S, Sun J, Chu T, Emr SD. ESCRTing proteins in the endocytic pathway. Trends Biochem Sci. 2007;32:561-73.

9. Hurley JH, Hanson PI. Membrane budding and scission by the ESCRT machinery: it's all in the neck. Nat Rev Mol Cell Biol. 2010;11:556-66.

10. Morshed S, Sharmin T, Ushimaru T. TORC1 regulates ESCRT-0 complex formation on the vacuolar membrane and microautophagy induction in yeast. Biochem Biophys Res Commun. 2020;522:88-94

11. Urbanowski JL, Piper RC. Ubiquitin sorts proteins into the intralumena degradative compartment of the late-endosome/vacuole. Traffic. 2001;2: 622-30

12. Chapman RE. Vacuolar sorting. Tracking down an elusive receptor. Curr Biol. 1994:4:1019-22.

13. MacDonald C, Payne JA, Aboian M, Smith W, Katzmann DJ, Piper RC. A family of tetraspans organizes cargo for sorting into multivesicular bodies. Dev Cell. 2015:33:328-42

14. Raymond CK, Howald-Stevenson I, Vater CA, Stevens TH. Morphological classification of the yeast vacuolar protein sorting mutants: evidence for a prevacuolar compartment in class E vps mutants. Mol Biol Cell. 1992;3:1389_ 402.

15. Rieder SE, Banta LM, Kohrer K, McCaffery JM, Emr SD. Multilamellar endosome-like compartment accumulates in the yeast vps28 vacuolar protein sorting mutant. Mol Biol Cell. 1996;7:985-99. 
16. Zhu L, Jorgensen JR, Li M, Chuang YS, Emr SD. ESCRTs function directly on the lysosome membrane to downregulate ubiquitinated lysosomal membrane proteins. elife. 2017;6:e26403.

17. Cowles CR, Odorizzi G, Payne GS, Emr SD. The AP-3 adaptor complex is essential for cargo-selective transport to the yeast vacuole. Cell. 1997;91: 109-18.

18. Stepp JD, Huang K, Lemmon SK. The yeast adaptor protein complex, AP-3, is essential for the efficient delivery of alkaline phosphatase by the alternate pathway to the vacuole. J Cell Biol. 1997;139:1761-74.

19. Dell'Angelica EC. AP-3-dependent trafficking and disease: the first decade. Curr Opin Cell Biol. 2009;21:552-9.

20. Reggiori F, Black MW, Pelham HR. Polar transmembrane domains target proteins to the interior of the yeast vacuole. Mol Biol Cell. 2000;11:3737-49.

21. Sun $B$, Chen $L$, Cao W, Roth AF, Davis NG. The yeast casein kinase Yck3p is palmitoylated, then sorted to the vacuolar membrane with AP-3-dependent recognition of a YXXPhi adaptin sorting signal. Mol Biol Cell. 2004;15: 1397-406.

22. Pokrzywa W, Guerriat B, Dodzian J, Morsomme P. Dual sorting of the Saccharomyces cerevisiae vacuolar protein Sna4p. Eukaryot Cell. 2009;8:278-86.

23. Wang YX, Catlett NL, Weisman LS. Vac8p, a vacuolar protein with armadillo repeats, functions in both vacuole inheritance and protein targeting from the cytoplasm to vacuole. J Cell Biol. 1998;140:1063-74.

24. Tang F, Peng Y, Nau JJ, Kauffman EJ, Weisman LS. Vac8p, an armadillo repeat protein, coordinates vacuole inheritance with multiple vacuolar processes. Traffic. 2006;7:1368-77.

25. McNally EK, Brett CL. The intralumenal fragment pathway mediates ESCRTindependent surface transporter down-regulation. Nat Commun. 2018;9: 5358.

26. Omari S, Makareeva E, Roberts-Pilgrim A, Mirigian L, Jarnik M, Ott C, Lippincott-Schwartz J, Leikin S. Noncanonical autophagy at ER exit sites regulates procollagen turnover. Proc Natl Acad Sci U S A. 2018;115: E10099-108.

27. Li WW, Li J, Bao JK. Microautophagy: lesser-known self-eating. Cell Mol Life Sci. 2012;69:1125-36

28. Sato M, Seki T, Konno A, Hirai H, Kurauchi Y, Hisatsune A, Katsuki H. Rapamycin activates mammalian microautophagy. J Pharmacol Sci. 2019; 140:201-4.

29. Mostofa MG, Rahman MA, Koike N, Yeasmin AM, Islam N, Waliullah TM, Hosoyamada S, Shimobayashi M, Kobayashi T, Hall MN, Ushimaru T. CLIP and cohibin separate rDNA from nucleolar proteins destined for degradation by nucleophagy. J Cell Biol. 2018;217:2675-90.

30. Sharmin T, Morshed S, Ushimaru T. PP2A promotes ESCRT-0 complex formation on vacuolar membranes and microautophagy induction after TORC1 inactivation. Biochem Biophys Res Commun. 2020:524:614-20.

31. Yeasmin AM, Waliullah TM, Kondo A, Kaneko A, Koike N, Ushimaru T. Orchestrated action of PP2A antagonizes Atg13 phosphorylation and promotes autophagy after the inactivation of TORC1. PLoS One. 2016;11: e0166636.

\section{Publisher's Note}

Springer Nature remains neutral with regard to jurisdictional claims in published maps and institutional affiliations.

Ready to submit your research? Choose BMC and benefit from:

- fast, convenient online submission

- thorough peer review by experienced researchers in your field

- rapid publication on acceptance

- support for research data, including large and complex data types

- gold Open Access which fosters wider collaboration and increased citations

- maximum visibility for your research: over $100 \mathrm{M}$ website views per year

At $\mathrm{BMC}$, research is always in progress.

Learn more biomedcentral.com/submissions 